Review

\title{
Study on Fiber-Optic Low-Coherence Interferometric and Fiber Bragg Grating Sensors
}

\author{
Yunjiang RAO \\ Key Laboratory of Optical Fiber Sensing \& Communications (Ministry of Education), University of Electronic Science \\ and Technology of China, Chengdu, 611731, China \\ *Corresponding author: Yujiang RAO_E-mail: yjrao@uestc.edu.cn
}

\begin{abstract}
This article reviews author's research work on fiber-optic sensors over the last twenty years. It includes two aspects: low-coherence interferometric sensors (LCI) and fiber Bragg grating (FBG) sensors. For LCI sensors, author's work mainly focuses on the interrogation and multiplexing methods for Fizeau and Fabry-Perot interferometric sensors at the University of Kent at Canterbury (UKC), UK, and study on novel Fabry-Perot interferometric sensors and their multiplexing methods at Chongqing University (CQU) and University of Electronic Science \& Technology of China (UESTC), China, respectively. For FBG sensors, a number of multiplexing schemes are proposed and demonstrated at $\mathrm{UKC}$, and then novel methods for realization of multi-parameter measurement and long-distance measurement based on the FBG sensor and its combination with other optical fiber sensors are also reported at CQU \& UESTC. Thus, author's study on these two topics can be divided into two periods, at UKC and at CQU \& UESTC, China. This review is presented in such a time sequence.
\end{abstract}

Keywords: Fiber optic sensors, low-coherence interferometry, fiber Bragg grating, Fabry-Perot

\section{Introduction}

Fiber-optic sensors have attracted great attention and have been extensively investigated in the last 30 years. They are playing increasingly important roles in civil engineering, military, aerospace, energy, transportation, chemical and biomedical science, etc. As a researcher in the field of fiber optics, I feel lucky to have worked from 1992 to 1999 in one of the most famous research centers in the field of fiber optic sensing - the Applied Optics Group led by Prof. David Jackson at the University of Kent at Canterbury (UKC), UK. I worked there as a (senior) research fellow, and engaged in the study of fiber-optic low-coherence interferometric sensors (LCI) and fiber Bragg grating (FBG) sensors for various applications, including strain, pressure, temperature, refractive index, and biomedical measurement, etc.

In 1999, I returned to Chongqing University (CQU), China, where I started to lead a research group and established a research lab focusing on fiber optics technology. In 2005, I joined the University of Electronic Science \& Technology of China (UESTC). After I returned to China, my research work on LCI and FBG sensors was significantly extended.

This paper presents a systematic review of my research activities during the two periods mentioned above. This paper is organized as follows. In Sections 2 and 3, main research results and achievements on LCI and FBG sensors are described,

Received: 9 July 2011 / Revised version: 6 August 2011

(C) The Author(s) 2011.This article is published with open access at Springerlink.com 
respectively, especially, work achieved in the last ten years. A summary is given in Section 4.

\section{Study on LCl sensors}

\subsection{Basis of LCI sensors}

Usually, a fiber-optic interferometric sensing arrangement using a broadband light source with a relatively small coherence length is called an LCI sensor system [1]. In implementing LCI, Fabry-Perot (F-P) interferometers are especially attractive due to the advantages of compact size, low cost and high precision. Normally, when an F-P cavity is much larger than the diameter of the fiber core, it is termed a Fizeau interferometer.

The cavity of F-P/Fizeau interferometer is formed between two parallel reflective surfaces, which when illuminated can generate an interferometric output. In sensing applications, the optical path difference (OPD) is set to maximize the output signal generated by phase changes induced by the measurand. The output of the interferometer can be interrogated by another interferometer with a similar OPD or by an optical spectrum analyzer (OSA), to determine the induced change in the OPD of the sensing interferometer.

It was demonstrated that LCI sensors based on F-P/Fizeau interferometers have much better wavelength stability compared with the sensor illuminated with a highly coherent laser diode, and its measurement accuracy is virtually insensitive to optical power fluctuations. It can therefore offer a much higher resolution $\left(>10^{4}: 1\right)$. It becomes an important technique for the absolute remote measurement of quasi-static parameters, such as displacement, temperature, pressure, strain, and refractive index [1].

In the first period of my research work (Section 2.2), most of our attention was focused on precise interrogation of the optical output signal generated by LCI sensors, The accuracy of identifying the position of the zero OPD was greatly enhanced by the invention of light source synthesizing method.
Also, the Fizeau-based LCI sensor systems were demonstrated for a range of pressure measurements with multiplexing capability, which have found practical applications. In the second period (Section 2.3), the F-P interferometric sensors based on photonic crystal fibers (PCFs), were fabricated by using the methods of arc-fusion, laser micromachining and chemical etching, giving birth to a new generation of micro fiber-optic F-P interferometers. These new devices can significantly improve the sensing performance of conventional F-P interferometric sensors and have great potential for mass-production with lower cost. Furthermore, we also proposed novel signal processing and multiplexing schemes to enable the sensing systems to be used for multi-parameter and quasi-distributed measurements.

\subsection{Study on LCI sensors at UKC}

\subsubsection{Source synthesizing for $\mathrm{LCl}$ interrogation}

In a system that uses a receiving interferometer to interrogate the sensing interferometer, the position of the central fringe is a key parameter used to determine the OPD of LCI sensors. For light sources with relatively small coherence length, the intensity difference between the central fringe and adjacent fringes may be too small to allow its position to be determined, seeing Fig. 1(c). Thus, a high signal-to-noise ratio (SNR) is required in order to identify the central fringe. To overcome this problem, we proposed a novel source synthesizing technique, i.e. the summation method [2]. This method is based on combining the autocorrelation functions of two broad band light sources with widely separated wavelengths. With this approach, the output intensity from the interferometer is the intensity superimposition of two sets of interference patterns. Thus, the amplitude difference between the central fringe and the adjacent fringes is obviously increased, as shown in Fig. 1(b), and the required minimum SNR can be reduced by as much as $30 \mathrm{~dB}$.

Later, we proposed an improved method, which 
synthesizes a light source with a dominant central fringe in its autocorrelation function by multiplying the autocorrelation functions of two low-coherence sources rather than summing them $[3,4]$. Figure 1(a) indicates that the output leads to a better identification of the central fringe.
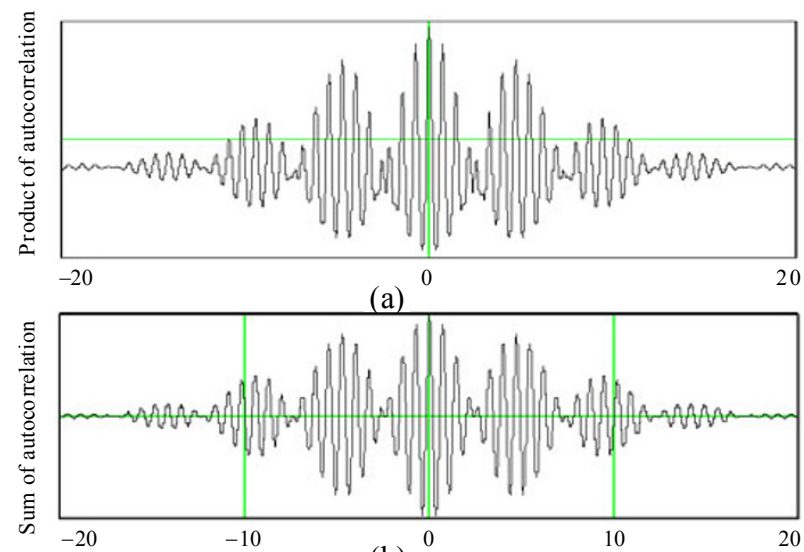

(b)

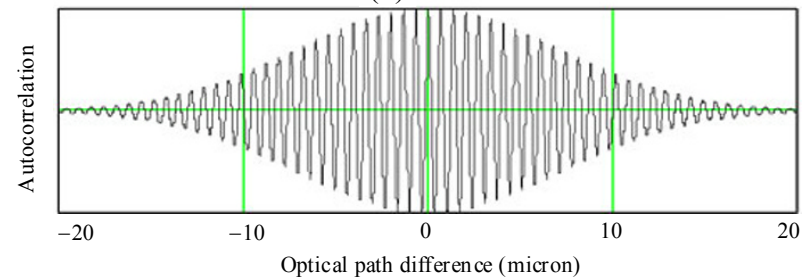

(c)

Fig. 1 Profiles of autocorrelation function: (a) product, (b) sum, and (c) normal [2,3].

The light source synthesizing method for LCI interrogation has been extensively developed by other research groups, such as the one at City University, UK.

\subsubsection{Pressure sensors based on LCI}

We have developed a series of pressure sensors based on F-P/Fizeau interferometers [5-10]. A typical experimental setup is given in Fig. 2. A Fizeau pressure sensor with temperature compensation and dual-wavelength coherence reading was developed [6]. A Fizeau interferometer is used to measure the pressure while another Fizeau interferometer with similar cavity length is arranged in parallel with the pressure sensor to compensate the temperature effect. A Michelson interferometer serves as the local receiving interferometer to interrogate the signal of the Fizeau interferometer.
The signal processing method is termed dual-wavelength coherence interferometry and uses two light sources with wavelengths of $\lambda_{1}$ and $\lambda_{2}$. Through this approach, the change of the OPD of the interferometric cavity is measured absolutely by determining the optical phase change of the scanned interferometric pattern, $\Phi_{1}$ (obtained from source 1), and the change of the optical phase difference between the interferometric signals, $\Delta \Phi$ (obtained from both sources). $\Phi_{1}$ is used for the high resolution measurement of the OPD while $\Delta \Phi$ is used for the determination of the interferometric fringe number, making absolute measurement feasible. This work was supported by an EPSRC/LINK project in collaboration with Queensgate Instruments, UK.

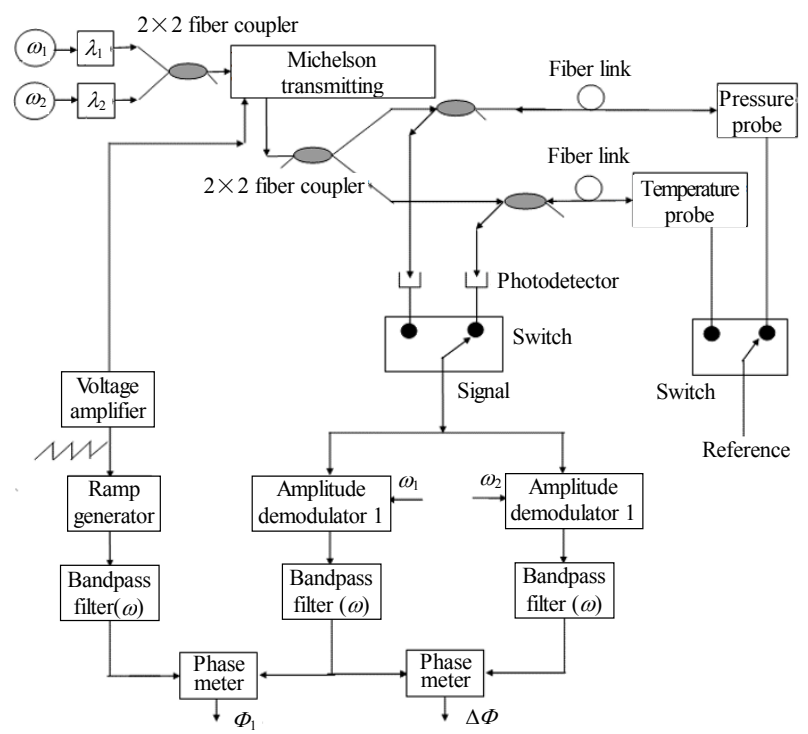

Fig. 2 Schematic diagram of a pressure sensor system $\left(\Phi_{1}\right.$ denotes absolute phase at $\lambda_{1}$, and $\Delta \Phi$ denotes differential phase between $\lambda_{1}$ and $\left.\lambda_{2}\right)[1,6]$.

Based on the technique mentioned above, 3 types of sensors including low pressure, medium pressure, high pressure, were developed for various applications [7-10]. Table 1 lists the performance of the "in-house" fabricated Fizeau sensors under different conditions of pressure.

Table 1 Performance of 3 types of pressure sensors.

\begin{tabular}{cccc}
\hline Pressure & Dynamic measurement range & Accuracy & Measurement range \\
\hline Low & $6.7 \times 10^{3}: 1$ & $\pm 1 \%$ & $0-0.48 \mathrm{bar}$ \\
Medium & $3.6 \times 10^{4}: 1$ & $\pm 0.15 \%$ & $0-10 \mathrm{bar}$ \\
High & $1.67 \times 10^{4}: 1$ & $\pm 0.69 \%$ & $0-1000 \mathrm{bar}$ \\
\hline
\end{tabular}




\subsection{Study on LCI sensors in China}

\subsubsection{New generation of micro F-P interferometers}

\section{A. PCF based F-P interferometers}

PCFs have shown their unique advantages when compared with traditional fibers in many application areas. Taking advantage of their unique characteristics in confining light, we reported novel PCF-based F-P interferometers for sensing of strain, refractive index and temperature [11-15], for the first time.

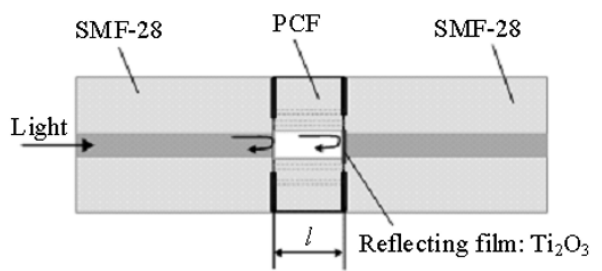

(a)

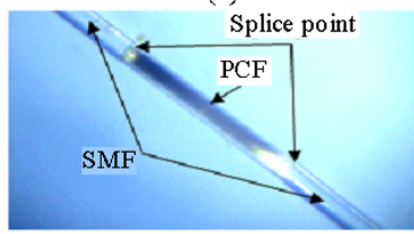

(b)

Fig. 3 A hollow-core PCF-based F-P sensor: (a) schematic of configuration and (b) photograph [11]

To measure the stress of engine blades, we designed a hollow-core PCF based F-P sensor which could survive high temperatures of up to $600{ }^{\circ} \mathrm{C}$ [11]. As shown in Fig. 3, this sensor is fabricated by splicing a section of hollow-core PCF between two standard single-mode fibers (SMFs). A thin film of $\mathrm{Ti}_{2} \mathrm{O}_{3}$ is coated at the distal fiber end to enhance the fringe contrast of the sensor. An improved design of such a sensor was reported lately [12]. Figure 4 shows the experimental results. We can see the output spectrum of the sensor is relatively strong and the fringes are clear. Moreover, the strain response of the sensor is relatively insensitive to temperature. This sensor has demonstrated a strain sensitivity of about $5.9 \mathrm{~nm} / \mu \varepsilon$, operating at high temperatures of up to $600{ }^{\circ} \mathrm{C}$.

To measure the change of refractive index, two schemes for the F-P interferometric sensors were developed. One was formed by splicing a section of endlessly PCF between two SMFs. The other was formed by cascading a section of hollow-core fiber and a normal PCF to an SMF [13, 14]. These two types of sensors have refractive index resolution of $2 \times 10^{-5}$ for glycerin solution and resolution of $1.6 \times 10^{-5}$ for air, respectively.

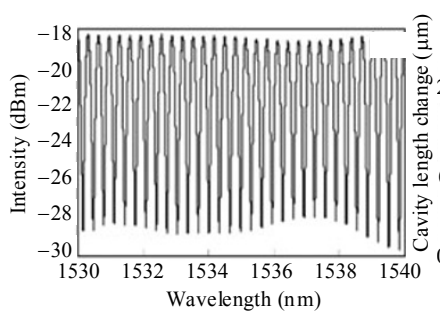

(a)

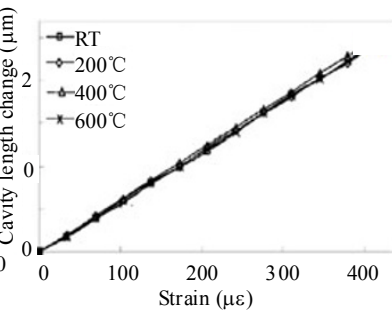

(b)
Fig. 4 (a) Output spectrum of a hollow-core PCF-based F-P sensor and (b) stain response of the sensor [12].

Recently, we developed a PCF-based F-P sensor with all-silica structure to measure temperature of up to $1200{ }^{\circ} \mathrm{C}$ [15]. The F-P sensor, as shown in Fig. 5(a), is fabricated by splicing a cleaved PCF (MM-HNA-5) to a SMF (SMF28e). The PCF has a small core diameter of $5.1 \mu \mathrm{m}$ surrounded by six holes with a diameter of $10.5 \mu \mathrm{m}$, seeing Fig. 5(b). The interface at the splice (mirror 1) and the end facet of the PCF eventually form the F-P cavity.

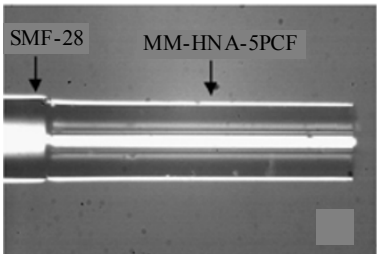

(a)

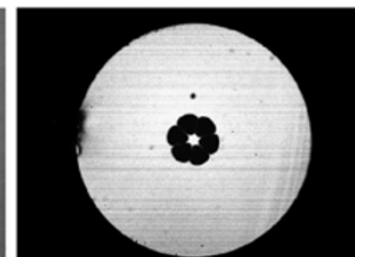

(b)
Fig. 5 (a) Photographs of the all-silica sensor and (b) the cross-section of the PCF [15].

Figure 6 shows the temperature response of the all-silica F-P sensor. In Fig.6(a), the quality of the fringes of output spectra at different temperatures is excellent. The temperature sensitivity of $17.5 \mathrm{~nm} /{ }^{\circ} \mathrm{C}$ is obtained. Figure 6(b) indicates that the sensor has a good linearity and a good repeatability over the whole measurement range (i.e., $20{ }^{\circ} \mathrm{C}-1200{ }^{\circ} \mathrm{C}$ ). 


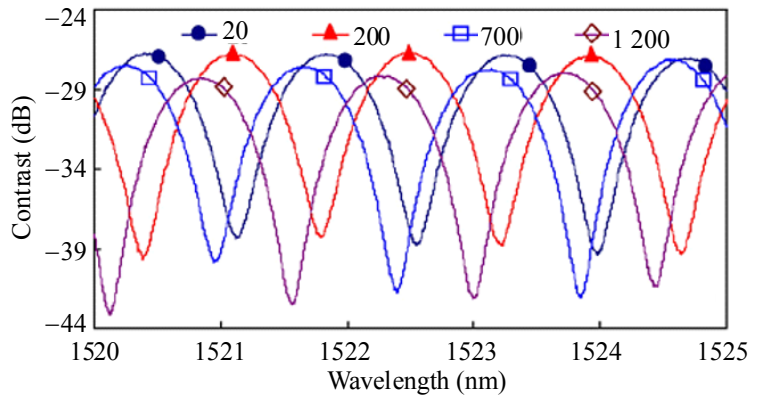

(a)

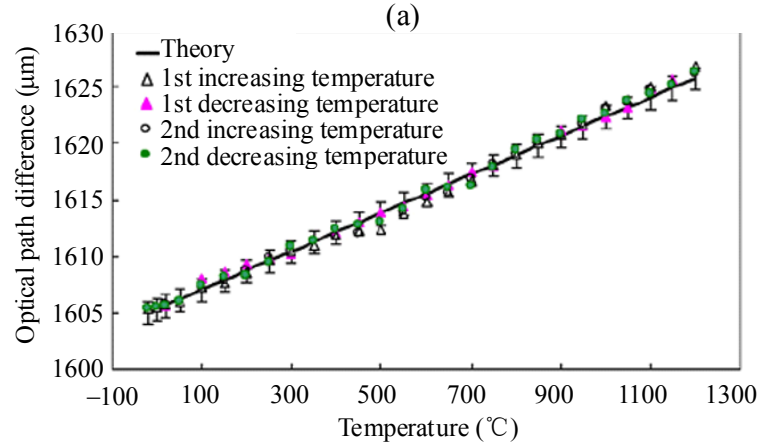

(b)

Fig. 6 (a) Output spectra of the all-silica sensor at different temperatures and (b) change of the optical path difference of the all-silica sensor [15].

\section{$B$. Laser micro-machined F-P interferometers}

Advanced micromachining methods based on a near-infrared femtosecond laser and a 157-nm excimer laser in the research center of Optical Fiber Technology at UESTC enable us to produce high quality F-P interferometers. Figures 7(a) and 7(b) show the photo of the 157-nm laser system in our center and a schematic of laser processing setup based on this system, respectively.

One type of F-P cavities fabricated was obtained by directly carving a micro-groove along an SMF or a PCF $[16,17]$, seeing Fig. 8(a). Another type was obtained by carving a micro-hole on one fiber end and then splicing it to another cleaved fiber [18-22], seeing Fig. 8(b). The signals of these sensors were interrogated by identifying the spectral shift of the interferometric fringes. Hence, high sensing accuracy was obtainable due to the high fringe contrast and good cavity finesse of these F-P cavities. In our experiments, these micro-machined F-P sensors were verified to have high sensitivity to various measurands, such as strain and refractive index. Moreover, they were insensitive to the variation of temperature and could work in high temperature environments.

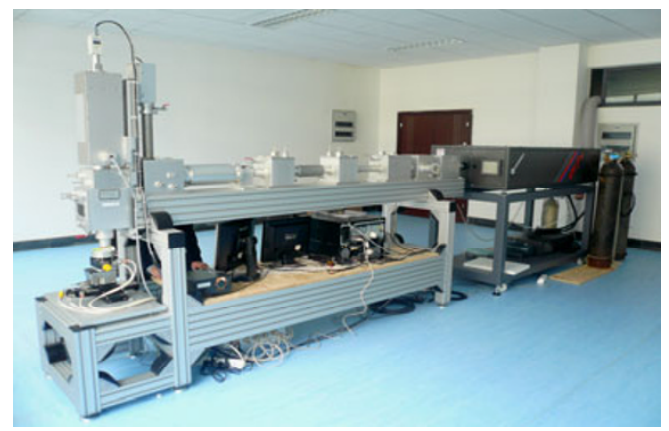

(a)

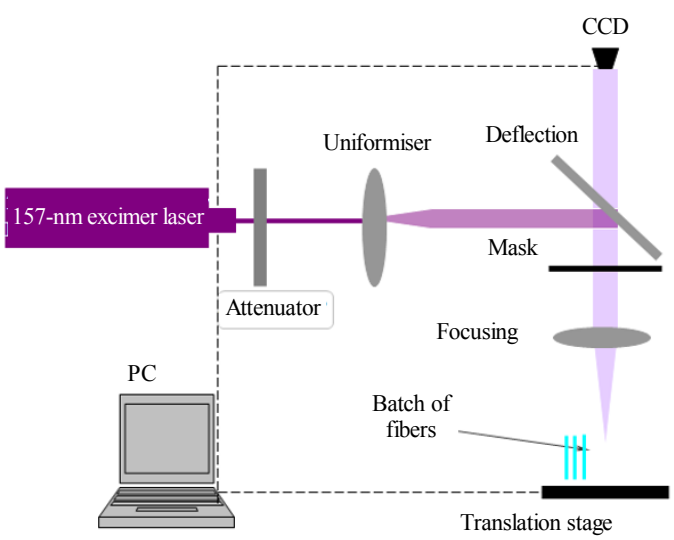

(b)

Fig. 7 (a) $157 \mathrm{~nm}$ excimer laser micro-machining system and (b) schematic of the processing setup.

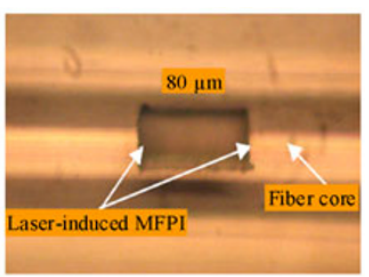

(a)

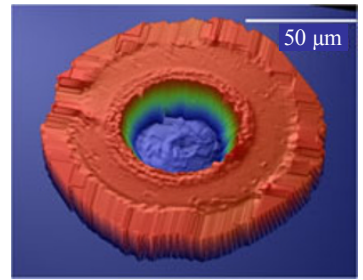

(b)
Fig. 8 (a) F-P cavity on an SMF and (b) micro-hole on a fiber end to be spliced with another fiber to form an F-P cavity [16, 18].

Figure 9 shows the reflective spectrum of an F-P interferometer micro-machined by a femtosecond laser on an SMF and PCF, respectively. In Fig. 9(a), it can be seen that the interferometric fringes are just good enough for sensing purposes, however, the fringe visibility is relatively low and is improved to $15 \mathrm{~dB}$ later by optimizing the parameters of the laser [16]. In Fig. 9(b), the fringe visibility for PCF F-P interferometer is improved by a few $\mathrm{dB}$ due to 
special characteristics of the PCF, i.e., the air-holes of the PCF can help the heat generated by the laser to be dispersed quicker than that of the SMF, leading to better quality in terms of fabrication of optical surfaces inside the fiber. The strain sensitivity and temperature sensitivity (i.e., wavelength change of the reflection dip) of the two types of sensors are given in Table 2. It is observed that the SMF sensor is more sensitive to strain than the PCF sensor, and both of the two types of sensors are relatively insensitive to temperature, i.e., their temperature sensitivity is 11 times smaller than that of the inline SMF etalon sensors reported previously [18].

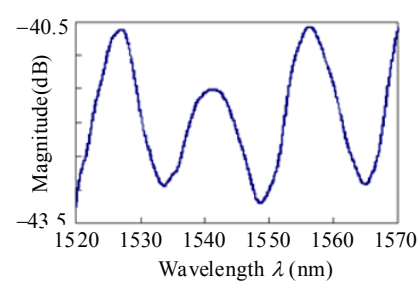

(a)

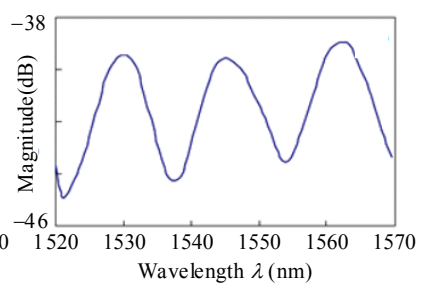

(b)
Fig. 9 Reflective spectrum of an F-P interferometer on the SMF [(a)] and the PCF [(b)], processed by a femtosecond laser [16].

Table 2 Sensitivity of the micro-machined sensors [16].

\begin{tabular}{ccc}
\hline Fiber type & Strain sensitivity & Temperature sensitivity \\
\hline $\mathrm{SMF}$ & $0.006 \mathrm{~nm} / \mu \varepsilon$ & $-0.002 \mathrm{~nm} /{ }^{\circ} \mathrm{C}$ \\
$\mathrm{PCF}$ & $0.0045 \mathrm{~nm} / \mu \varepsilon$ & $-0.0021 \mathrm{~nm} /{ }^{\circ} \mathrm{C}$ \\
\hline
\end{tabular}

Based on the progress achieved with the F-P interferometer micro-machined with a femtosecond laser, an F-P strain sensor was micro-machined on a PCF using a 157-nm laser as this laser could offer much better micromachining quality due to better absorption of the laser energy at $157 \mathrm{~nm}$ by the fiber. The obtained fringe contrast is about $26 \mathrm{~dB}$, as shown in Fig. 10 [17] and highlighted by Photonics Spectra in December 2007 [19].

The most dominant characteristic of these micro sensors is that they can maintain their high performance virtually constant for high temperature strain measurements up to $800{ }^{\circ} \mathrm{C}$, seeing Fig. 11(a). Figure 11(b) gives the variation of the cavity length as a function of temperature. It can be seen that total change is just $\pm 10 \mathrm{~nm}$ over $800{ }^{\circ} \mathrm{C}$ range.

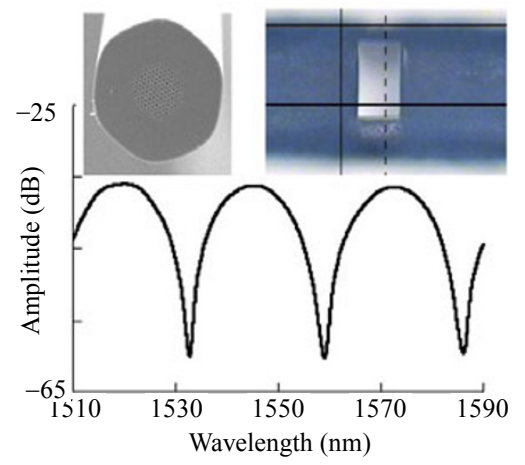

Fig. 10 Reflective spectrum of a micro F-P sensor curved on a PCF (the inset shows photos of cross-section of the PCF and the micro F-P sensor [17].

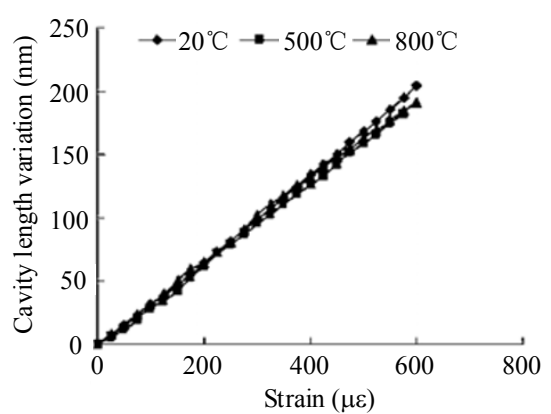

(a)

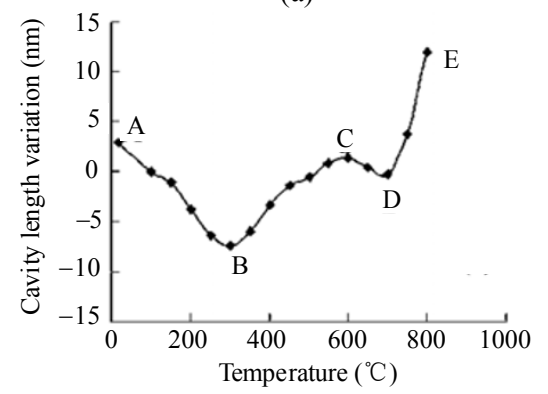

(b)

Fig. 11 Strain response $[(a)]$ and temperature response [(b)] of the F-P sensor curved on a PCF [17].

Another type of F-P interferometers was realized by carving a micro-hole on one fiber end and then splicing it to another cleaved fiber [20-24], by using the $157-\mathrm{nm}$ micromachining method shown in Fig. 7(b). We have enhanced the fringe contrast of the F-P sensor to about $30 \mathrm{~dB}$ [21], seeing Fig. 12 (a). From Figs. 12 (b) and 12(c), it is found that the cavity length has a very linear relationship with the strain applied, even at high temperature. Moreover, the temperature sensitivity of such a type of sensors is low and only $0.0006 \mathrm{~nm} /{ }^{\circ} \mathrm{C}$. 


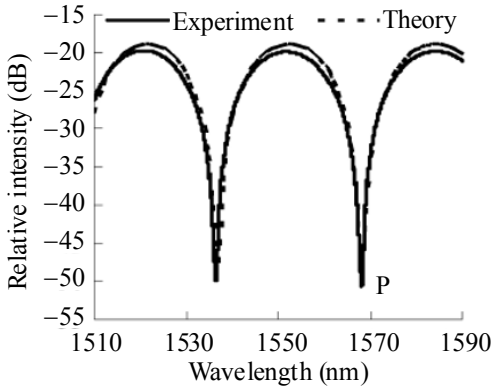

(a)

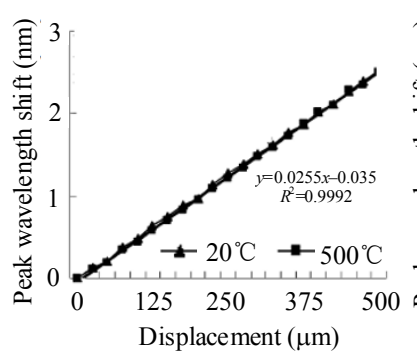

(b)

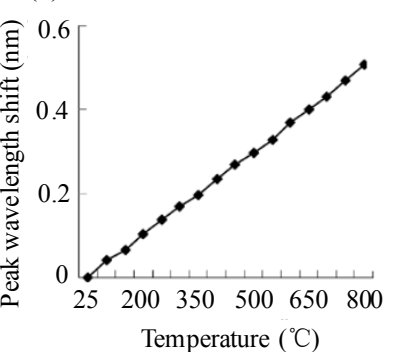

(c)
Fig. 12 (a) Reflective spectrum of an improved F-P sensor fabricated in the same way as that shown in Fig. 8(b), (b) strain response, and (c) temperature response of the F-P sensor [21].

Based on the strain sensor, a precise pressure sensor with similar structure was developed by cutting and polishing the fiber end-surface to a thickness of several microns which could be used as the diaphragm of the pressure sensor. An accuracy of $\pm 0.1 \%$ was achieved. The photo of this pressure sensor is displayed in Fig. 13.

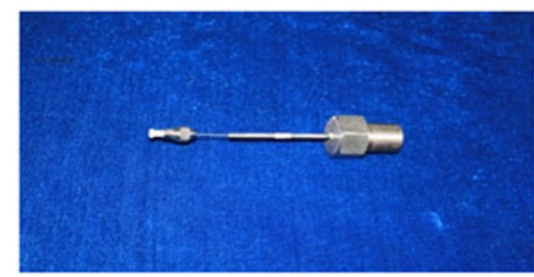

Fig. 13 Photo of the micro-machined F-P pressure sensor.

Furthermore, high temperature F-P sensors based on sapphire and silica fibers were fabricated by the laser micromachining method and demonstrated. This work was presented at the Twenty-first International Conference on Optical Fiber Sensors (OFS-21[25]). This type of sensor can operate at high temperatures of up to $1100{ }^{\circ} \mathrm{C}$ with a temperature sensitivity of about $50 \mathrm{pm} /{ }^{\circ} \mathrm{C}$, as shown in Fig. 14, which is about 5 times higher than that of the purely silica F-P sensor.

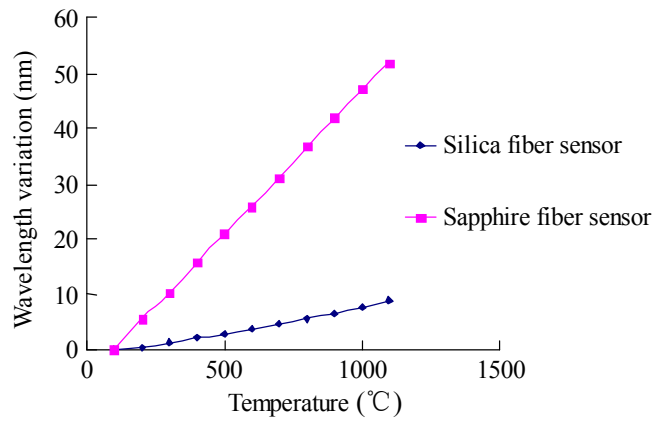

Fig. 14 Temperature responses of sapphire and silica F-P sensors.

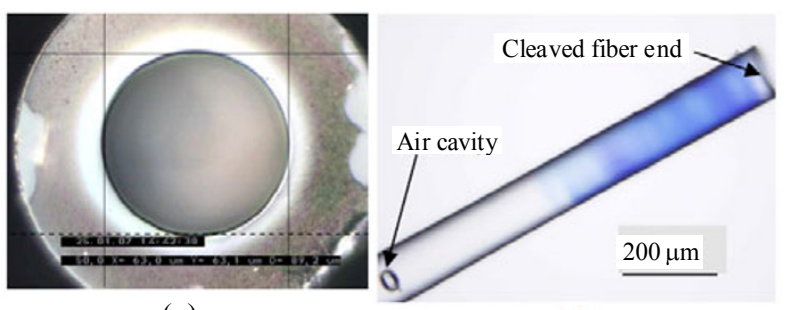

(a)

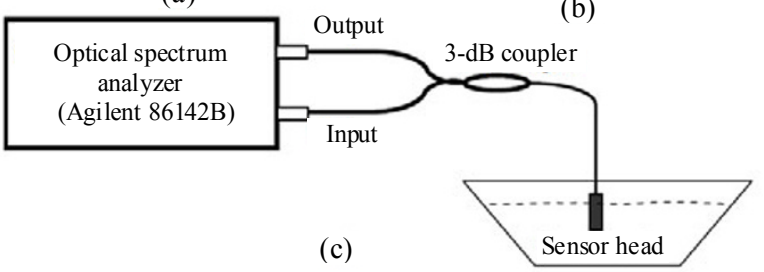

Fig. 15 (a) Microscopic image of the micro-machined hole introduced on the fiber cross section, (b) microscopic image of the fabricated sensor head, and (c) experimental setup for refractive-index sensing [20].

Based on an "enclosed cavity inside the fiber" structure, a novel dual-parameter sensor based on dual F-P interferometers was proposed for temperature-independent measurement of refractive index. The microscopic image of the sensor and the experimental setup are given in Fig. 15, respectively. The external refractive index is determined according to the maximum fringe contrast (obtained by locating the minimum dip, $\lambda_{1}$, and the adjacent peak, $\lambda_{2}$ ) of the interference fringes, seeing Fig. 16(a). A sensor of this type can provide temperature-independent measurement of practically any refractive index larger than that of air and offers a refractive-index resolution of $4 \times 10^{-5}$ (fringe contrast resolution is $0.001 \mathrm{~dB}$ ) in its linear operating range, seeing Figs. 16(b) and 16(c). 


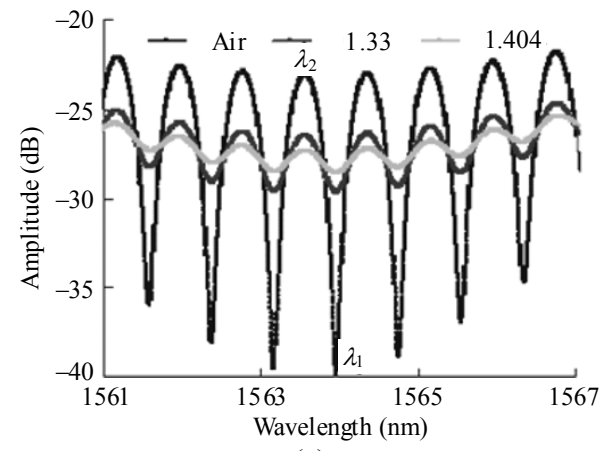

(a)

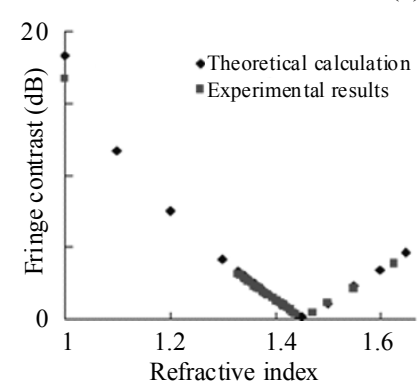

(b)

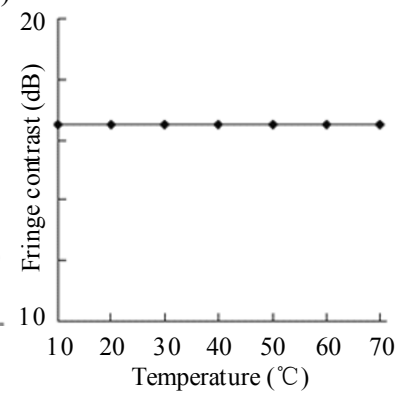

(c)
Fig. 16 (a) Reflection spectrum of the sensor, (b) variation of the fringe contrast with the refractive index, and (c) variation of the fringe contrast measured in air with the temperature [22].

For multi-parameter measurement, we demonstrated a hybrid fiber-optic sensor consisting of a micro-machined F-P interferometer and a long-period fiber grating (LPFG) [24]. The experimental setup is shown in Fig. 17. Both reflection and transmission measurements are performed to monitor the outputs of the F-P sensor and the LPFG, respectively, by using a high-accuracy OSA.

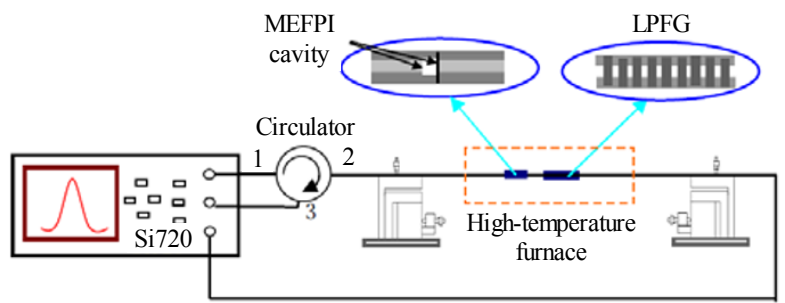

Fig. 17 Experimental setup of the hybrid LPFG and micro extrinsic F-P interferometric (MEFPI) sensor [24].

The reflective spectrum of the F-P sensor is displayed in Fig. 18(a). It is seen that an excellent visibility of about $30 \mathrm{~dB}$ is achieved. The transmitted light passes through the F-P cavity and then enters into the LPFG. The mixed (transmitted) spectrum of the F-P sensor and the LPFG is shown in Fig. 18(b). It is seen that the signal of the LPFG is much stronger than that of the F-P cavity. It is known that the dip position of the reflective spectrum of the F-P cavity is sensitive (insensitive) to strain (temperature) and the spectrum of LPFG is sensitive to temperature, so the former and latter can be used to measure strain and temperature, respectively, and the sensing performance is shown in Table 3.
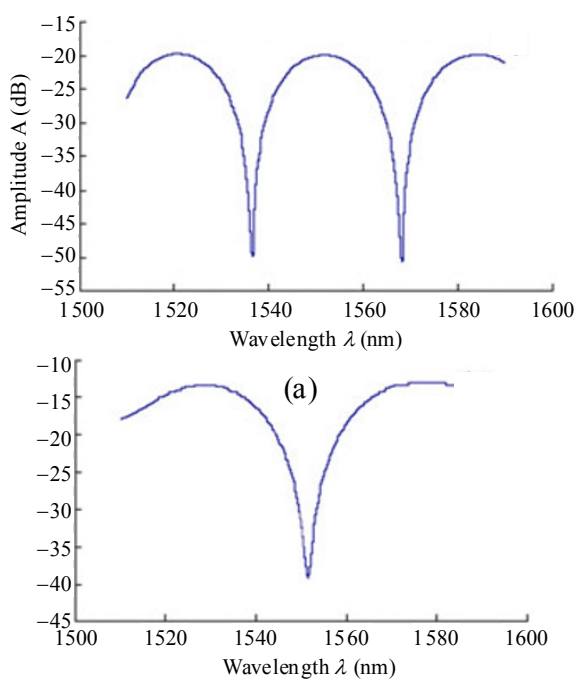

(b)

Fig. 18 (a) Reflective spectrum of the F-P sensor and (b) mixed (transmitted) spectrum of the F-P sensor and the LPFG [24].

Table 3 Performance of the hybrid sensor.

\begin{tabular}{ccc}
\hline Measurand & Resolution & Measurement range \\
\hline Strain & $0.5 \mu \varepsilon\left(\right.$ at $\left.500{ }^{\circ} \mathrm{C}\right)$ & $0-1000 \mu \varepsilon$ \\
Temperature & $0.02{ }^{\circ} \mathrm{C}$ & $0-700{ }^{\circ} \mathrm{C}$ \\
\hline
\end{tabular}

\section{Chemically etched F-P interferometers}

Another research interest is to fabricate F-P interferometric sensors by using a chemical etching method [26-30]. The etching method is simple and cost effective.

Figure 19 shows the fabrication process of a micro F-P interferometric sensor [26]. An Er-doped fiber (EDF) is etched by mixed hydrochloric and hydrofluoric acid. Because of Er doping, the etching rate of the core is three times greater than that of the cladding, so the etched hole can be deep enough to 
form an F-P cavity when it is spliced to an SMF, seeing Fig. 19(d). Such an etched F-P interferometric sensor can have high fringe contrast that is comparable to laser micro-machined F-P interferometric sensors. As shown in Fig. 20, the maximum visibility of the reflective spectrum is about $24 \mathrm{~dB}$, and the strain sensitivity is about $3 \mathrm{pm} / \mu \varepsilon$.

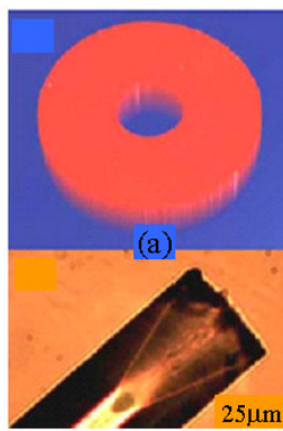

(c)

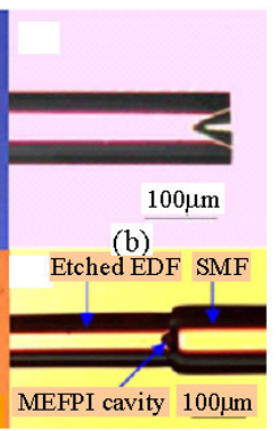

(d)
Fig. 19 (a) 3D profile of the etched EDF, (b) and (c) images of the fiber ends with etching depths of $50 \mu \mathrm{m}$ and $104 \mu \mathrm{m}$, respectively, and (d) MEFPI sensor [26].

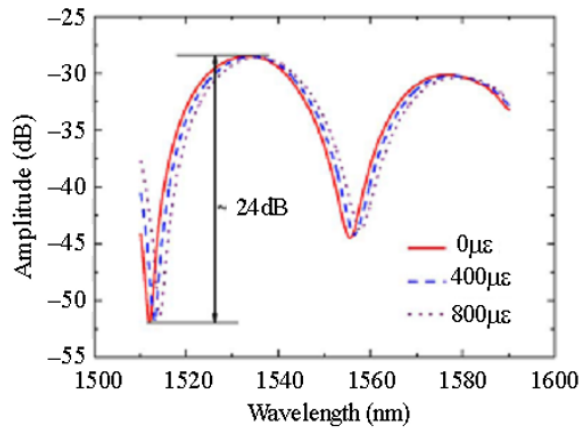

Fig. 20 Reflection spectra of the etched F-P interferometric sensor under different strains [26].

Recently, we proposed a novel etched F-P interferometric sensor for multi-parameter measurement $[27,28]$. Similar etching methods were used except that the fiber to be etched was a graded-index multimode fiber rather than an Er-doped fiber, as shown in the inset of Fig. 21. The periodic focusing effect of graded-index multimode fiber can be used to enhance the sensing performance. It is demonstrated that the sensitivities for refractive index and temperature are $45.05 \mathrm{~dB} / \mathrm{RIU}$ and $11.5 \mathrm{pm} /{ }^{\circ} \mathrm{C}$, respectively.

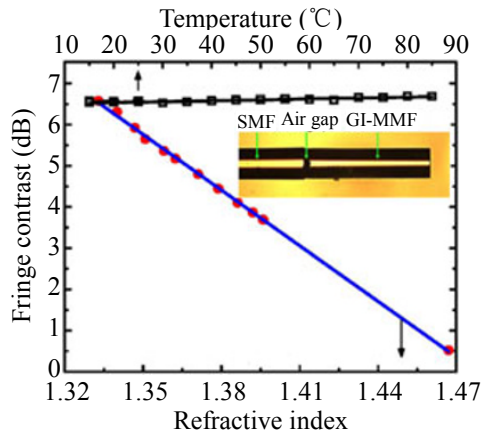

Fig. 21 Refractive index and temperature responses of the F-P sensor (the inset shows the sensor formed between a SMF and an etched graded-index multimode fiber (GI-MMF)) [27].

\subsubsection{Multiplexing of F-P/Fizeau sensors}

Besides the design of the above mentioned point sensors, we also proposed multiplexing schemes to promote their system-level applications. Special efforts were focused on Fizeau sensors as their relative long cavity length enabled more sensors to be multiplexed. In our studies, methods based on wavelength division multiplexing (WDM), spatial frequency division multiplexing (SFDM), time division multiplexing (TDM), and their combinations were demonstrated [31-35].

\section{A. SFDM of Fizeau sensors}

The basis of SFDM is that the reflective spectra of Fizeau sensors with different cavity lengths can be distinguished through fast Fourier transform (FFT) transformation. Figure 22 shows schematically an SFDM system of Fizeau sensors proposed. In our experiment, eleven Fizeau sensors with different cavity lengths $(0.5 \mathrm{~mm}, 1 \mathrm{~mm}, \ldots, 6 \mathrm{~mm})$ were multiplexed. The reflected signals of the Fizeau sensors were amplified by an erbium-doped fiber amplifier (EDFA) and then detected by an OSA. The mixed spectrum of the eleven sensors is shown in Fig. 23(a). Correspondingly, Fig. 23(b) shows the transformed spectrum by using high-pass filtering and FFT. Obviously, the component corresponding to each cavity length can be distinguished. Multiplexing of up to eleven Fizeau sensors was experimentally achieved. 


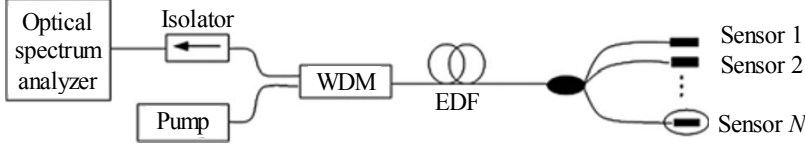

Fig. 22 SFDM of fiber-optic Fizeau sensor system based on optical amplification [32].

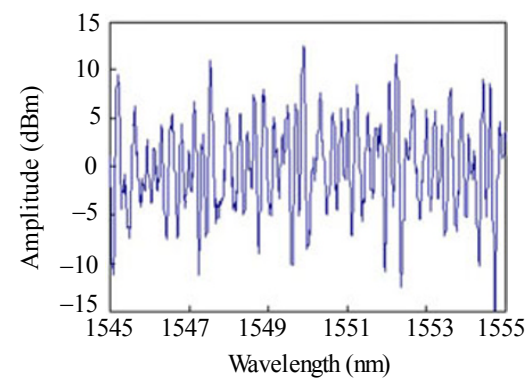

(a)

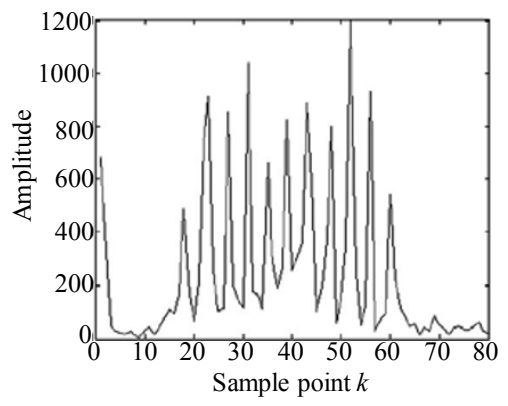

(b)

Fig. 23 (a) Mixed signal of 11 Fizeau sensors and (b) Fourier transform spectrum of 11 signals [35].

\section{B. Combination of SFDM and WDM}

By using coarse WDM (CWDM), the spectrum of a broadband light source can be divided into different wavelength channels. An SFDM was used in each wavelength channel to further increase the number multiplexed.

The multiplexing system based on the combination of SFDM and CWDM is shown schematically in Fig. 24 [32]. The CWDM divides light into four channels. Within each WDM channel, several (four in our experiment) Fizeau sensors with different cavity lengths are located. Figure 25(a) shows that the signals from WDM channels 1 and 2 are clearly separated for their different regions of wavelength. Figure 25(b) indicates that the four signals from the same WDM channel are also separated clearly due to different cavity lengths of the corresponding sensors.

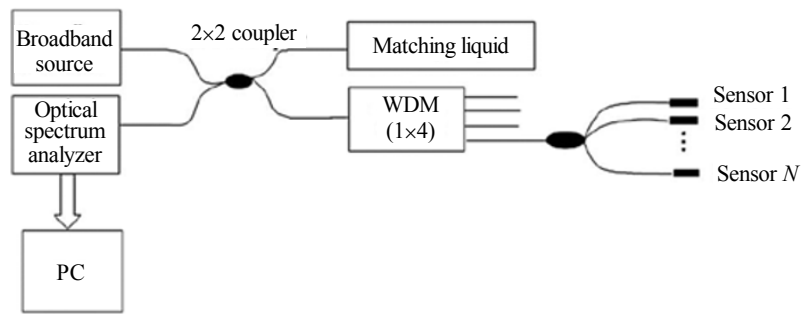

Fig. 24 Schematic diagram of the hybrid SFDM/WDM multiplexing system [35].

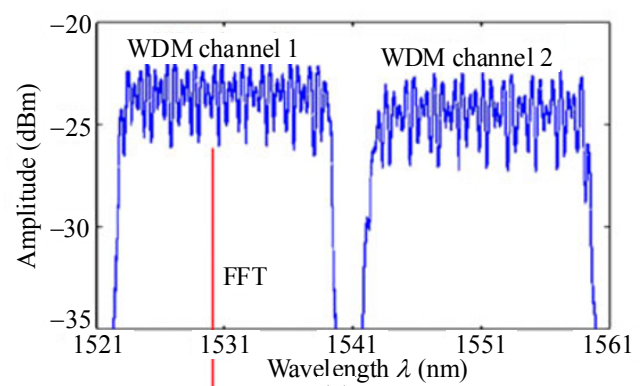

(a)

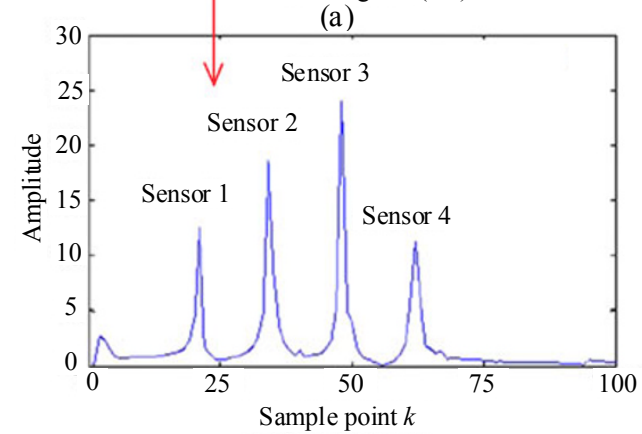

(b)

Fig. 25 (a) Mixed signal for channels 1 and 2, and (b) Fourier transform spectrum of channel 1 [35].

\subsubsection{Industrial applications of F-P sensors}

A typical practical application for our F-P sensors is strain measurement of a stress balance in a high-temperature environment, which is of great importance for aerospace testing.

Figure 26 shows the photograph of the stress balance. The high-temperature F-P strain sensors micro-machined using a 157-nm laser were attached to the beams of the balance to measure the six parameters of the stress balance, i.e., strength and moment in the $x, y$ and $z$ directions, seeing Fig. 26(b). The sensitivity is $0.0036 \mathrm{rad} / \mu \varepsilon$, the linearity is $99.991 \%$, and the tolerable environmental temperature is above $300{ }^{\circ} \mathrm{C}$. 

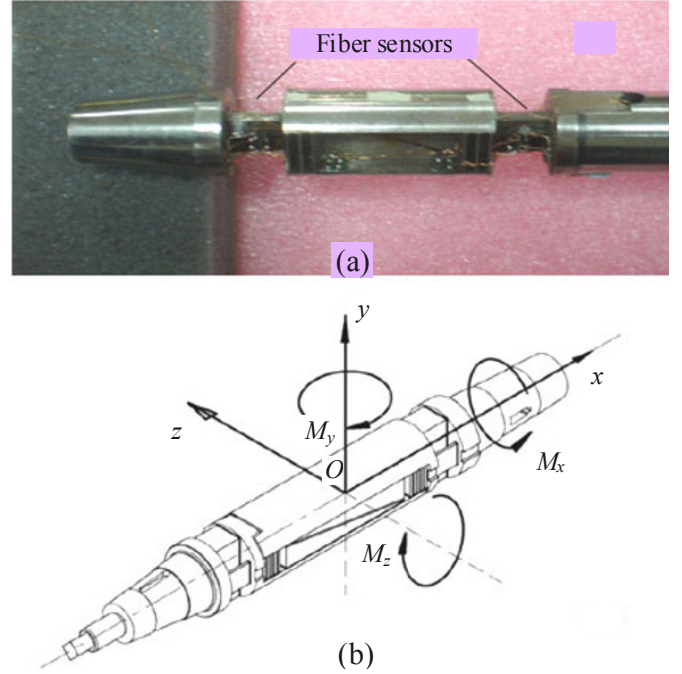

(b)

Fig. 26 (a) Photograph of the strain balance and (b) strength and moment directions of the strain balance.

\section{Fiber Bragg grating sensors}

\subsection{Basis of FBG sensors}

An FBG has a periodic variation of refractive index in the fiber core. It reflects a particular wavelength (the Bragg wavelength) of light and transmits all others with low losses. The Bragg wavelength, $\lambda_{\mathrm{B}}$, is given by $[36,37]$

$$
\lambda_{B}=2 n \Lambda
$$

where $n$ is the effective index and $\Lambda$ is the grating period. The value of $\lambda_{\mathrm{B}}$ is very sensitive to stain and temperature. Studies on FBG sensors are flourishing due to their distinct advantages when compared with FOIS, i.e., insensitive to the power fluctuations of the light source, compact structure, easy fabrication and also easy to multiplex for quasi-distributed sensing.

During the first period of my research (Section 3.2), improved interrogation methods were used to enhance the performance of FBG sensing systems. Special attentions were focused on multiplexed FBG sensing systems and their applications in medical measurement. During the second period (Section 3.3), we introduced FBGs in the fabrication of F-P and Fizeau cavities to design high quality sensors. Meanwhile, integrated sensing systems based on FBGs were proposed for long-distance and dynamic measurements. A few field applications of the FBG sensing system were also discussed.

\subsection{Study on FBG sensors at UKC}

\subsubsection{Dual-cavity interferometric interrogation}

The wavelength shift of FBG sensors can be detected with a scanning interferometer. For an interferometric detection scheme, there is a trade off between resolution and range of measurements. We have proposed a dual-cavity interferometric interrogation scheme to increase the measurement range whilst maintaining the high resolution [38].

The experimental setup is described in Fig. 27. We can see that two sets of interferometric fringes are obtained by stepping the scan interferometer from a long cavity (range 1) to a short cavity (range 2). The optical phase output from the short cavity gives a high-resolution measurement while the phase output from the long cavity determines the number of fringes. This scheme allows a measurement range of up to $10^{5}: 1$, which is $1-2$ order higher than the conventional interferometric detection scheme.

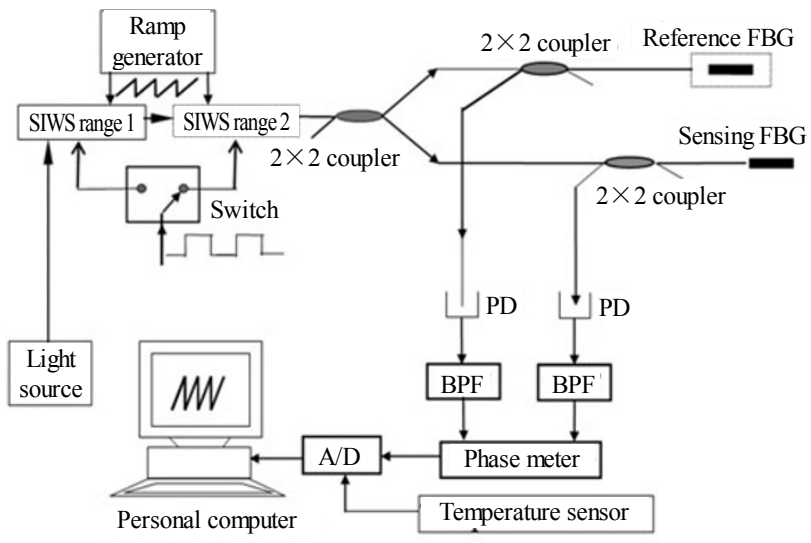

Fig. 27 Schematic of the dual-cavity interferometric scanning scheme (SIWS: stepped Michelson interferometric wavelength scanner; BPF: bandpass filter; PD: photodiode; A/D: analogue-to-digital convertor) [36, 38]

An improved version of this scheme using two cascaded interferometers that used frequency division multiplexing was demonstrated for real-time strain measurement and a range to 
resolution of $4 \times 10^{4}: 1$ was achieved [39].

\subsubsection{FBG-based F-P sensors}

Although the method mentioned above can achieve both large dynamic range and high resolution simultaneously, it requires the wavelengths of the two sources to be highly stabilized, which is relatively difficult to be realized in practice. To overcome this disadvantage, we have proposed a novel inline F-P interferometric sensor formed by two FBGs with the same central wavelength [40]. The basic concept is shown in Fig. 28. Such an FBG-based F-P interferometer is mainly used to obtain ultrahigh resolution strain measurement via phase-change detection of the F-P interferometric signal, $\Delta \Phi$, while the wavelength-shift of the two FBGs, $\Delta \lambda$, is used for determining the fringe number, $N$, of the interferometric signal.

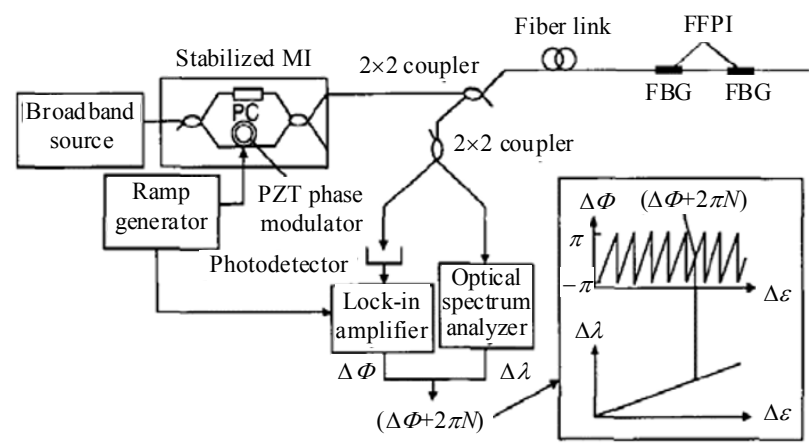

Fig. 28 Schematic of the FBG based F-P sensor system [40].

The inset of Fig. 28 indicates that $\Delta \lambda$ gives a coarse reading for the applied strain and then $N$ is determined with this reading because the strain has a fixed relationship with the fringe number. Hence, the total absolute phase change, $\Delta \Phi+2 N \pi$, as well as the OPD is determined. Using the similar idea, we formed a Fizeau cavity between an FBG and a highly reflective surface to measure temperature (via the FBG) and displacement (via the Fizeau cavity) simultaneously [41].

\subsubsection{Multiplexing of FBG sensors}

We have proposed a series of multiplexing techniques for FBG sensors based on SDM, WDM,
TDM, and their combinations [42-50]. Special efforts were under taken to improve the multiplexing capacity and SNR as well as to reduce the cross-talk of the system. These systems were also extended for the use of 2D/3D quasi-distributed network and in vivo medical applications.

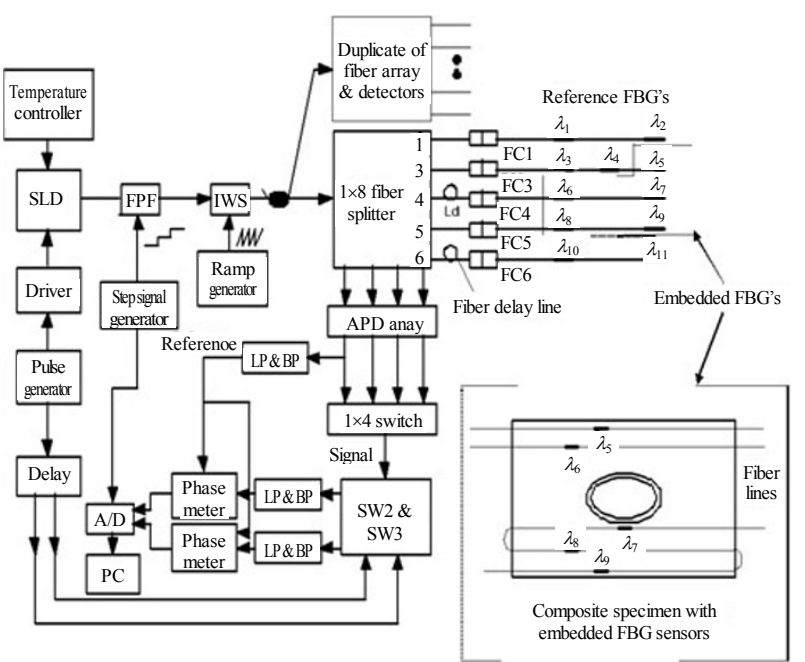

Fig. 29 Schematic of the SDM + WDM + TDM system (SLD: superluminescent diode; FPF: tunable F-P filter; IWS: interferometric wavelength scanner; APD: avalanche photodetector; BP: bandpass filter; LP: low-pass filter; SW: switch) [36].

One example of FBG sensing system that combines all the three multiplexing techniques is given in Fig. 29 [44, 45]. Both the tunable F-P filter (FPF) and the interferometric wavelength scanner (IWS) are combined in tandem and are located immediately after the broadband source rather than in front of the detector to allow all the FBG elements with similar wavelengths on all the fiber sensing channels to be interrogated simultaneously. The FPF is used to select specified FBG elements with different center wavelengths along a single fiber, whereas the IWS is used to achieve high-resolution wavelength-shift measurement by precisely detecting the phase change induced by the wavelength shift of the FBG. Using this system, static strain measurement of a modern composite material specimen with embedded FBGs (the inset of Fig. 29) was demonstrated. This scheme is capable of multiplexing a large number of FBGs 
$(>100)$ with strain resolution of about $1 \mu \varepsilon \mathrm{Hz}-1 / 2$.

To overcome the cross interactions between measurands of FBG sensors, we developed an FBG sensor system based on a combined SDM and WDM topology. The schematic of the system is presented in Fig. 30.

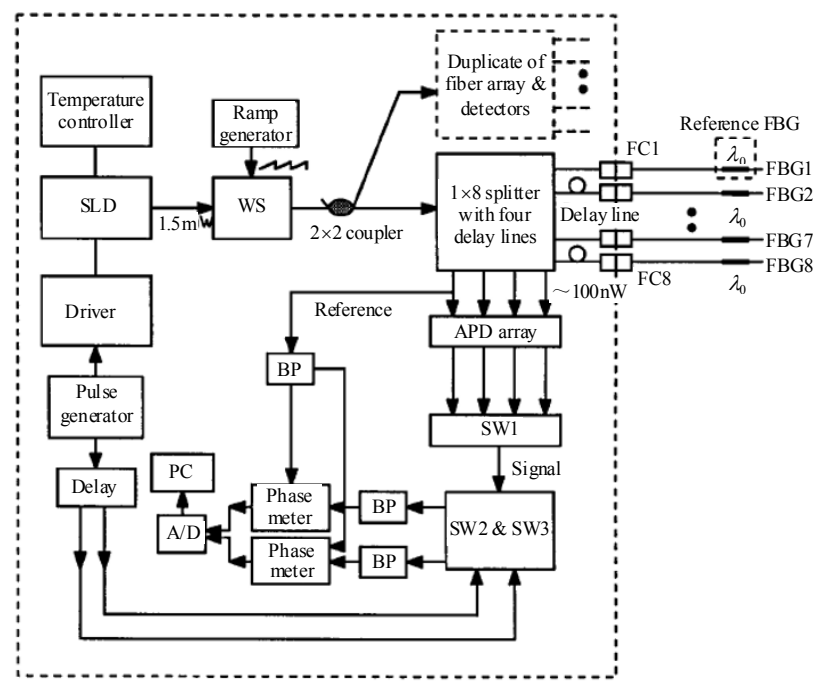

Fig. 30 Schematic of the SDM + WDM system for strain measurement with temperature compensation [43].

There were four output ports of the $1 \times 8$ fiber-optic splitter, with each port containing two fiber transmission lines with differential delay lengths (the time delay is $400 \mathrm{~ns}$ ). FBG sensors were spliced to the ends of these fiber lines. Eight FBG's were deployed in this network. It would be feasible to interrogate another eight FBG's by duplicating part of the network without reduction in the signal-to-noise ratio of each sensor, as also indicated in Fig. 30. The pulsed light was launched into the wavelength scanner (WS) and the $1 \times 8$ splitter. The return pulse signals from the FBG sensors were coupled back into the splitter and detected by an array of four avalanche photodetectors (APD). The signals from the APD array were selected by a switch (SW1). Thus each APD receives the returned signals from two FBG's separated in time by $400 \mathrm{~ns}$. These two signals were separated by two high-speed switches (SW2 and SW3). The phase information contained in the interference signal was recovered by the pseudo-heterodyne technique.
In the system, the reference FBG was deployed strain free and located in the same temperature environment as the sensing FBG's. Hence, a quasi-static strain sensing system with temperature compensation is realized. The detected signals from this eight-element FBG array are shown in Fig. 31(a). To show the delayed pulses clearly, we adjusted the amplitudes of these pulses by changing the joint loss within the fiber couplers. The de-multiplexed signals corresponding to four FBG sensors are shown in Fig. 31(b). Cross talk between two adjacent TDM channels was measured to be less than $-36 \mathrm{~dB}$. Similar systems were also proposed by us for simultaneously strain and temperature measurement [48].

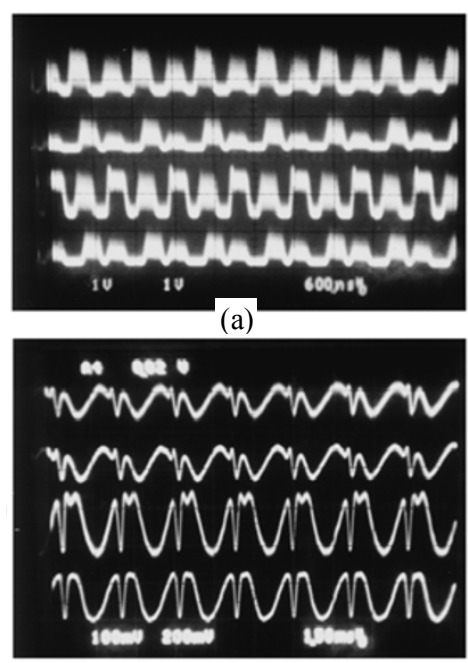

(b)

Fig. 31 Experimental waveforms: (a) photo detector return pulse signals for eight gratings (each trace corresponds to the signals from two gratings with TDM) and (b) demultiplexed signals from four gratings [43].

\subsection{Study on FBG sensors in China}

\subsubsection{Cascaded FBG/F-P sensors}

A novel sensor based on cascaded FBG/F-P interferometer (Fig. 32) is demonstrated for simultaneous measurement of the strain and temperature of advanced 3D braided composite materials [51]. Figure 33 shows the reflective spectra for both the FBG and the F-P interferometers. It can be seen that the signal amplitude of the FBG 
is much higher than that of the F-P interferometer, while the spectral width of the FBG is smaller than that of the F-P interferometer. Hence, the central wavelength of the FBG can be used for high accuracy temperature measurement, while the absolute cavity length change of the F-P interferometer can be used to measure the strain.

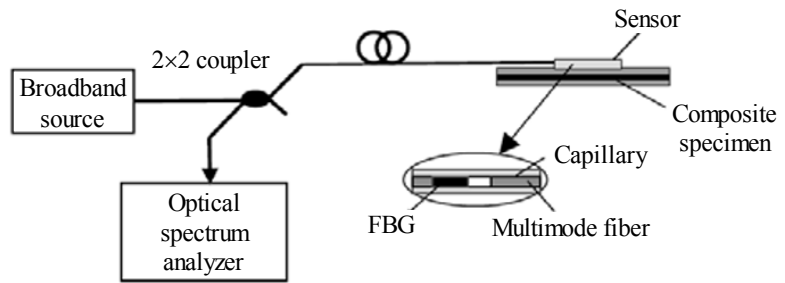

Fig. 32 Schematic diagram of the tandem FBG/F-P sensor system [51].

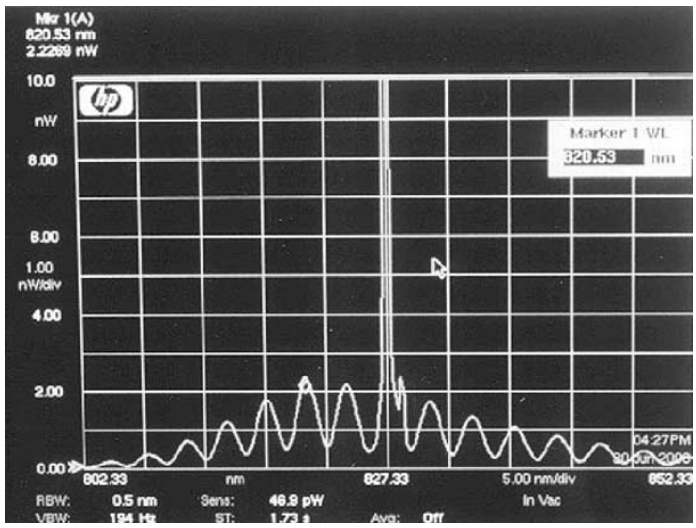

Fig. 33 Reflective spectra for both the FBG and the F-P interferometers [51].

\subsubsection{Multiplexing of FBG-based F-P sensors}

To enhance the number of FBG-based F-P sensors multiplexed for use in dynamic strain monitoring of large engineering structures, the F-P sensors formed by a chirped FBG pair are proposed and demonstrated, as shown in the inset of Fig. 34 [52]. WDM combined with SFDM is used to achieve a multiplexing capability of up to 1000 sensors. Figure 34 illustrates the schematic diagram of this multiplexing system. Light from a broadband light source is launched into a $2 \times M$ coupler and sent into $M$ fiber lines. In each fiber line, there are $N$ sensors formed by a series of chirped FBG pairs with different central wavelengths. Thus, the $N$ sensors have different wavelength domains and can be multiplexed via WDM. For the sensors within the same wavelength domain but in different fiber lines, they have different cavity lengths, namely, different spatial frequencies. Hence, they can be multiplexed via SFDM.

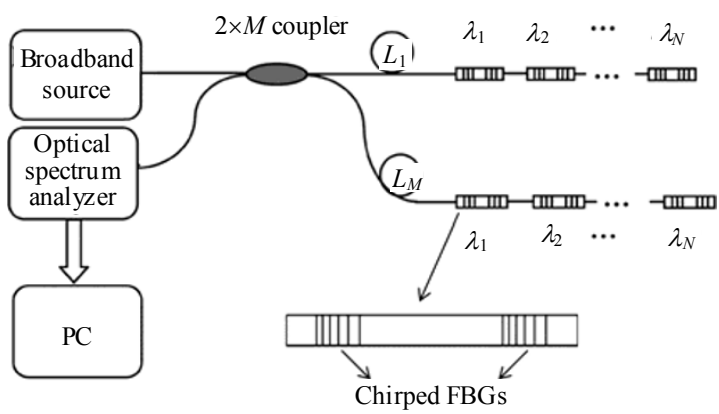

Fig. 34 Hybrid SFDM/WDM system for F-P sensors formed by chirped FBG pairs [52].

\subsubsection{Long-distance FBG sensing systems}

For long-distance FBG sensing systems, the maximum transmission distance with a broadband light source is normally limited to $25 \mathrm{~km}$ or so because of backscattering and intrinsic loss of the fiber link. We proposed an approach using a tunable fiber ring laser with hybrid erbium-doped fiber (EDF) and Raman amplification to achieve an optical SNR of more than $60 \mathrm{~dB}$ after $50-\mathrm{km}$ transmission [53].

The configuration of the $50-\mathrm{km}$ sensing system is shown in Fig. 35. The sensing FBGs with different central wavelengths are divided into two groups arranged at regions of $0-25 \mathrm{~km}$ and $25 \mathrm{~km}-50 \mathrm{~km}$. There is a Raman amplifier (pumped at $1455 \mathrm{~nm}$ ) and two EDFAs. The local EDFA generates amplified spontaneous emission (ASE) to illuminate the tunable filter and sensing FBGs. It also amplifies feedback signals from the FBGs. The narrowband ASE light tuned by the filter is sent into a $50-\mathrm{km}$ fiber link via a 90/10 coupler and a circulator. Lasing is generated as follows. First the reflected light from a specified FBG is amplified by both EDF amplification and Raman amplification through the indicated paths as one round trip in Fig. 35. Then, 
after multiple circulations in the fiber ring, the reflected light is re-amplified by multiple circulations and stabilized gradually, thus generating laser light. In addition, the multiplexing capacity of such a system can be largely exploited by using SDM with a $1 \times N$ coupler.

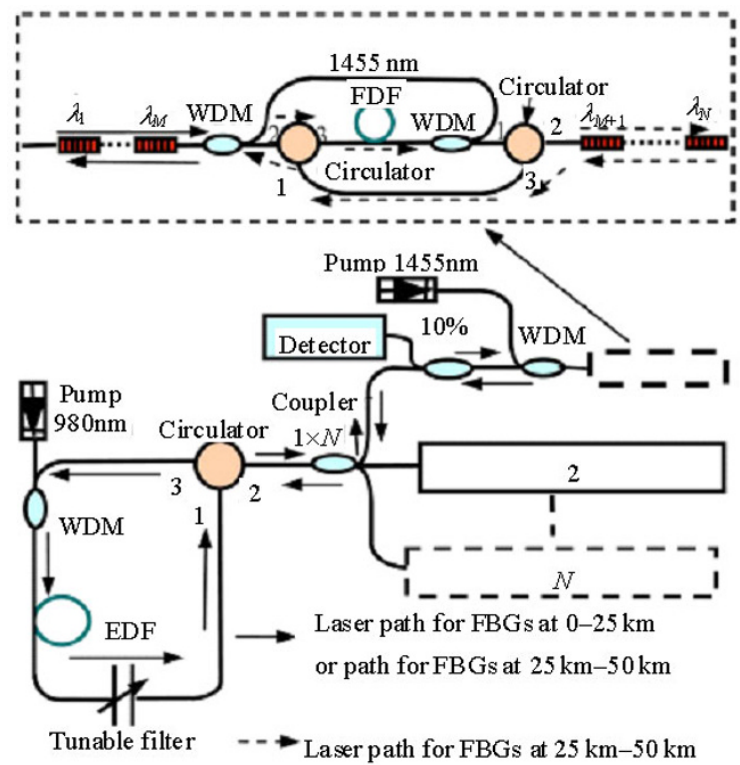

Fig. 35 Schematic of the 50-km FBG sensing system [53].

For demonstration, four FBGs with different wavelengths of $1544 \mathrm{~nm}, 1548 \mathrm{~nm}, 1552 \mathrm{~nm}$, and $1556 \mathrm{~nm}$ were used in the experiment. The results are show in Fig. 36. We can see that through hybrid EDF and Raman amplification, the SNRs of the two FBGs deployed at $50 \mathrm{~km}$ can be significantly enhanced, i.e., their SNRs are increased about $40 \mathrm{~dB}$.

Recently, we proposed an improved method based on hybrid EDF and Raman amplification to increase the transmission distance of the FBG sensing system to $300 \mathrm{~km}$ with an optical SNR of $4 \mathrm{~dB}$ [54]. To our knowledge, this is the longest inline sensing distance reported to date. The experimental setup is given in Fig. 37. Light from a tunable laser is modulated by an acoustic-optic modulator (AOM). The modulated light is amplified by an EDFA, and then split into two branches. The output light through the $1 \%$ branch is detected directly by a photo-detector PD1 as a reference signal. Light transmitted via the other branch is coupled into the FBG through a circulator and a WDM. The WDM is used to filter out the residual Raman pump laser power and related noise. Backward Raman amplification is employed here with a pump laser at $1480 \mathrm{~nm}$. PD2 is used to detect light reflected from the FBG.

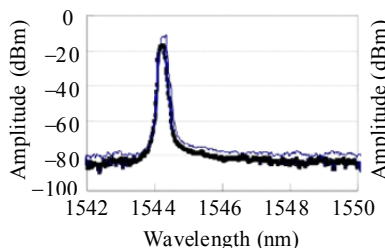

(a)

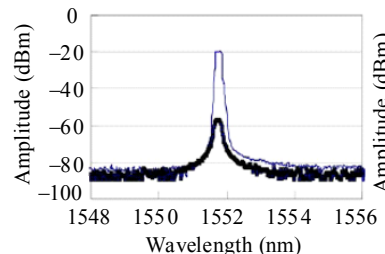

(c)

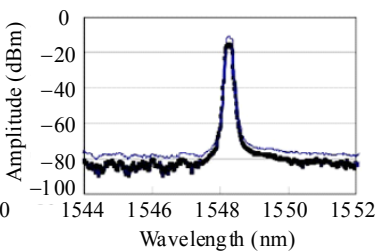

(b)

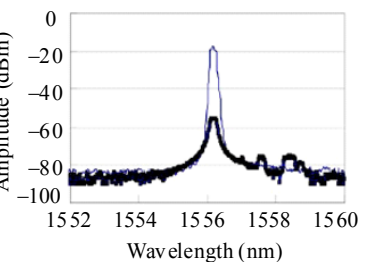

(d)
Fig. 36 Spectra of four reflective FBG signals with (thin curves) and without (thick curves) hybrid EDF and Raman amplification: (a) $1544 \mathrm{~nm}$ at $25 \mathrm{~km}$, (b) $1548 \mathrm{~nm}$ at $25 \mathrm{~km}$, (c) $1552 \mathrm{~nm}$ at $50 \mathrm{~km}$, and (d) $1556 \mathrm{~nm}$ at $50 \mathrm{~km} \mathrm{[53]}$

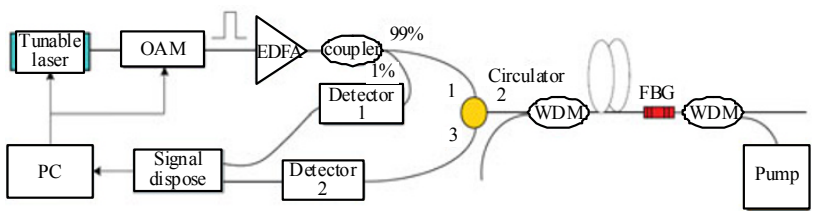

Fig. 37 Schematic of the 300-km FBG sensing system [54].

Figure 38 shows the value of SNR as a function of transmission distance of the systems. Although the SNR is reduced continuously with the distance, a usable SNR of about $4 \mathrm{~dB}$ is still achieved at the distance of $300 \mathrm{~km}$. The measurement accuracy of the static strain and temperature can remain constant.

For long-distance FBG sensing systems, we also used a Mach-Zehnder interferometer formed by two cascaded long-period fiber gratings serving as "many" multi-channel edge filters to detect the wavelength shift of FBG sensors, and high optical SNR of more than $60 \mathrm{~dB}$ was achieved. In addition, a spectral analyzing algorithm called "music modern spectrum estimation" was adapted to further 
improve the sensing resolution. The experimental results showed that a dynamic strain resolution of $0.1 \mu \varepsilon \mathrm{Hz}^{-1 / 2}$ at $700 \mathrm{~Hz}$ was obtained [55]

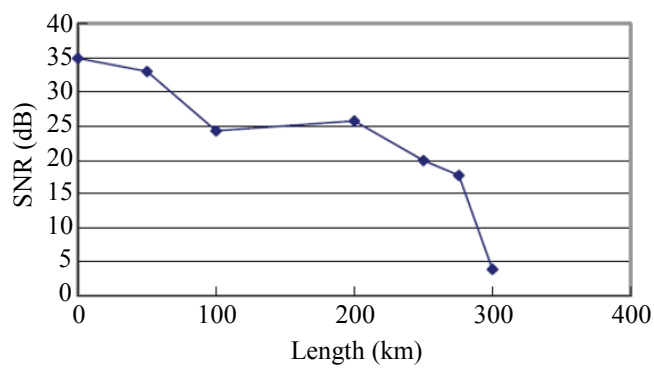

Fig. 38 SNR versus transmission distance of the $300-\mathrm{km}$ FBG sensing system [54].

\subsubsection{Field trials of FBG sensing systems}

The FBG-based fiber-optic fence is one of the typical examples for industrialization of our research results. Figure 39(b) shows the configuration of such an FBG-based fiber-optic fence used for intrusion monitoring [56].

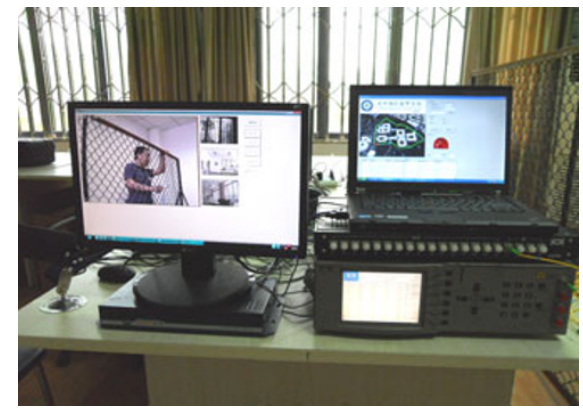

(a)

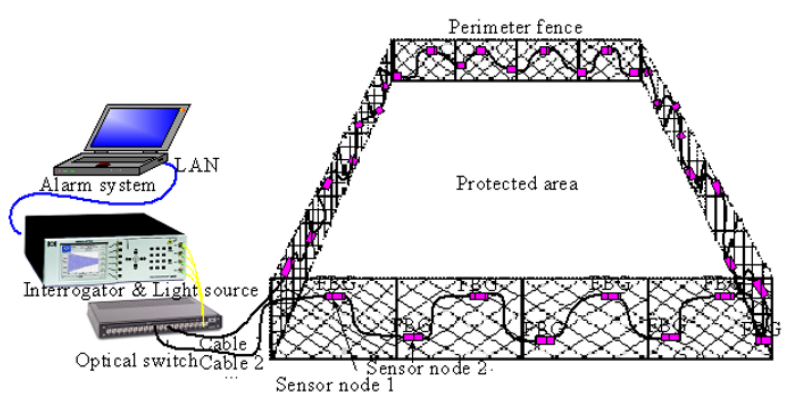

(b)

Fig. 39 Photo [(a)] and schematic [(b)] of a remote FBG-based fiber-optic fence intrusion detection system [56].

The system includes three parts: (1) the FBG sensing segments, connected in tandem along fiber cables, are attached to a physical fence to measure both the vibration and mechanical deformation (strain) of the fence; (2) the FBG interrogator is used to demodulate the feedback signals from the FBG arrays; (3) the alarm system (software) is the key part of this application and used for data processing to determine whether there are any threat as well as its mode and location. For the alarm system, an autocorrelation method is adapted, which enables us to detect extremely weak intrusions and even multiple intrusion events occuring. Experimental results indicate that the probability of detection of this system is higher than $99.5 \%$ with a spatial resolution of $5 \mathrm{~m}-10 \mathrm{~m}$.

Furthermore, FBG settlement sensors are developed at UESTC recently, as shown in Fig.40, and will be installed in the field for the real-time ground settlement monitoring of the high speed trains from Chongqing to Suining.

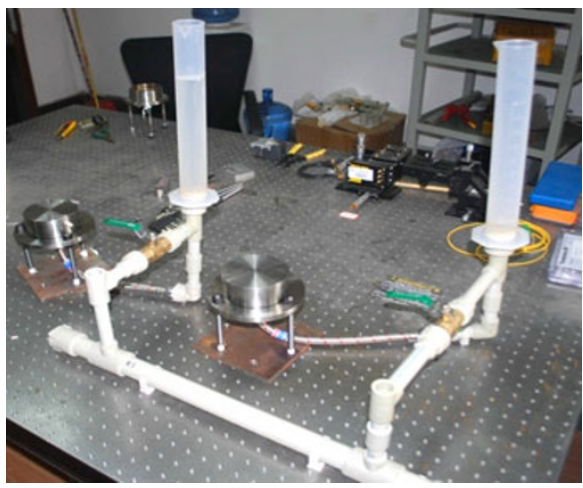

Fig. 40 Photo of FBG settlement sensors.

\section{Summary}

My research work on LCI and FBG sensors during the two periods at University of Kent and in China has been reviewed in this article. For both LCI and FBG sensors, I focused my efforts on finding advanced methods for design and fabrication of novel sensors, interrogation of sensing signals, and multiplexing of sensors.

For LCI sensors, we made important contributions to F-P/Fizeau type LCI sensors and systems. The light source synthesizing method proposed enabled the central fringe of the output to be identified with a significant reduction in required SNR. A series of Fizeau sensors with temperature 
compensation were developed for measurements of low, medium, and high-pressure. The so-called new generation F-P sensors based on PCFs, laser micro-machining and etching of normal fibers or PCFs have shown their unique capability in measuring refractive index, strain, pressure and temperature. The all-silica PCF-based F-P sensor can measure high temperature of up to $1200{ }^{\circ} \mathrm{C}$, and the laser micro-machined F-P strain sensor can operate at a high temperature of $800{ }^{\circ} \mathrm{C}$, while keeping a relatively high strain measurement accuracy of $\pm 1 \%$ without any temperature compensation. Compared with traditional sensors, the laser micro-machining technique overcomes the drawbacks of manual operation, such as relatively poor reproducibility and imprecise cavity length control, while the chemical etching technique provides us with a simple and economical way for fabricating micro optical fiber F-P sensors. We also proposed a number of advanced multiplexing schemes as well as demodulation methods to form sensing networks with these novel LCI sensors used for quasi-distributed and multi-parameter measurements.

For FBG sensors, we were among the earliest researchers on this topic in early 1990s. We proposed a number of topologies combining WDM, TDM and SDM to multiplex more than 100 sensors for static strain measurement of modern composite materials. The capability of FBG sensing systems was greatly enhanced to more than 1000 by using the novel F-P sensors formed by chirped FBG-pairs. On the other hand, we demonstrated long-distance FBG sensing system by using hybrid EDFA and Rama amplification. Transmission distances of $50 \mathrm{~km}, 100 \mathrm{~km}$ and $300 \mathrm{~km}$ were reported, respectively. The 300-km sensing distance remains the world record.

We are also pleased that some of our research achievements were or to be commercialized. Examples include the micro F-P sensors for high temperature applications, FBG-based fiber-optic fence intrusion detection systems, and FBG settlement sensors for the real-time ground settlement monitoring of the high speed trains. It is believed that with the more collaboration between our group and the industry, more fiber optic sensors will be commercialized.

\section{Acknowledgement}

The author would like to acknowledge Prof. David Jackson for his guidance, the author's team members at University of Electronic Science \& Technology of China and Chongqing University, and the support of Education Ministry of China and National Science Foundation of China.

Open Access This article is distributed under the terms of the Creative Commons Attribution License which permits any use, distribution, and reproduction in any medium, provided the original author(s) and source are credited.

\section{Reference}

[1] Y. J. Rao and D. A. Jackson, "Recent progress in fiber optic low-coherence interferometry," Meas. Sci. Technol., vol. 7, no. 7, pp. 981-999, 1996.

[2] Y. J. Rao, Y. N. Ning, and D. A. Jackson, "Synthesized source for white-light sensing systems," Opt. Lett., vol. 18, no. 6, pp. 462-464, 1993.

[3] Y. J. Rao and D. A. Jackson, "Improved synthesized source for white light interferometry," Electron. Lett., vol. 30, no. 17, pp. 1440-1441, 1994.

[4] Y. J. Rao and D. A. Jackson, "Long-distance fiber-optic white light displacement sensing system using a source-synthesizing technique," Electron. Lett., vol. 31, no. 4, pp. 310-312, 1995.

[5] Y. J. Rao and D. A. Jackson, "Prototype fiber-optic-based pressure probe with built-in temperature compensation with signal recovery by coherence reading," Appl. Opt., vol. 32, no 34, pp. 7110-7113, 1993.

[6] Y. J. Rao and D. A. Jackson, "Prototype fiber-optic-based ultrahigh pressure remote sensor using dual-wavelength coherence reading," Electron. Lett., vol. 29, no. 24, pp. 2142-2143, 1993.

[7] Y. J. Rao and D. A. Jackson, "Prototype fiber-optic-based ultrahigh pressure remote sensor with built-in temperature compensation," Rev. Sci. Instrum., vol. 65, no. 5, pp. 1695-1698, 1994. 
[8] Y. J. Rao and D. A. Jackson, "Prototype fiber-optic-based fizeau medical pressure and temperature sensor system using coherence reading," Meas. Sci. and Technol., vol. 5, no. 6, pp. 741-746, 1994.

[9] Y. J. Rao, D. A. Jackson, R. Jones, and C. Shannon, "Development of prototype fiber-optic-based Fizeau pressure sensors with temperature compensation and signal recovery by coherence reading," J. Lightwave Technol., vol. 12, no. 9, pp. 1685-1695, 1994.

[10] Y. J. Rao and D. A. Jackson, "Universal fiber-optic point sensor system for quasi-static absolute measurements of multiparameters exploiting low coherence interrogation," J. Lightwave Technol., vol. 14, no. 4, pp. 592-600, 1996.

[11] Y. J. Rao, T. Zhu, X. C. Yang, and D. W. Duan, "In-line fiber-optic etalon formed by hollow-core photonic crystal fiber," Opt. Lett., vol. 32, no. 18, pp. 2662-2664, 2007.

[12] Y. J. Rao, M. Deng, and T. Zhu, and H. Li, "In-line Fabry-Perot etalons based on hollow-corephotonic bandgap fibers for high-temperature applications," $J$. Lightwave Technol., vol. 27, no. 19, pp. 4360-4365, 2009.

[13] Y. J. Rao, M. Deng, D. W. Duan, and T. Zhu, "In-line fiber Fabry-Perot refractive-index tip sensor based on endlessly photonic crystal fiber," Sens. Actuators, A: Physical, vol. 148, no. 1, pp. 33-38, 2008.

[14] M. Deng,C. P. Tang, T. Zhu, Y. J. Rao, L. C. Xu, and M. Han, "Refractive index measurement using photonic crystal fiber-based Fabry-Perot interferometer," Appl. Opt., vol. 49, no. 9, pp. 1593-1598, 2010.

[15] T. Zhu, T. Ke, Y. J. Rao, and K. S. Chiang, "Fabry-Perot optical fiber tip sensor for high temperature measurement," Opt. Commun., vol. 283, no. 19, pp 3683-3685, 2010.

[16] Y. J. Rao, M. Deng, D. W. Duan, X. C. Yang, T. Zhu, and G. H. Cheng, "Micro Fabry-Perot interferometers in silica fibers machined by femtosecond laser," Opt. Express, vol. 15, no. 21, pp. 14123-14128, 2007.

[17] Z. L. Ran, Y. J. Rao, H. Y. Deng, and X. Liao, "Miniature in-line photonic crystal fiber etalon fabricated by $157 \mathrm{~nm}$ laser micromachining," Opt. Lett., vol. 32, no. 21, pp. 3071-3073, 2007.

[18] J. Sirks, T. A. Berkoff, R. T. Jones, H. Singh, A. D. Kersey, E. J. Friebele, and M. A. Putnam, "In-line fiber etalon (ILFE) fiber-optic ssensors," $J$. Lightwave Technol., vol. 13, no. 7, pp. 1256-1262, 1995

[19] B. Hitz, "To boldly go where no sensor has gone before," Photonics Spectra, no. 12, 2007.

[20] Z. L. Ran, Y. J. Rao, W. J. Liu, X. Liao, and K. S. Chiang, "Laser-micromachined Fabry-Perot optical fiber tip sensor for high-resolution temperature-independent measurement of refractive index," Opt. Express, vol. 16, no. 3, pp. 2252-2263, 2008.
[21] Z. L. Ran, Y. J. Rao, X. Liao, and H. Y. Deng, "Self-enclosed all-fiber in-line etalon strain sensor micromachined by 157-nm laser pulses," $J$. Lightwave Technol., vol. 27, no. 15, pp. 3143-3149, 2009.

[22] Y. J. Rao, M. Deng, T. Zhu, Q. T. Tang, and, G. H. Cheng, "Micromachining of an in-fiber extrinsic fabry-perot interfereometric (MEFPI) sensor by using a femtosecond laser," Key Eng. Mater, vol. 364-366 II, pp. 1203-1206, 2008.

[23] Z. L. Ran, Y. J. Rao, J. Zhang, Z. W. Liu, and B. Xu, "A miniature fiber-optic refractive-index sensor based on laser-machined Fabry-Perot interferometer tip," J. Lightwave Technol., vol. 27, no. 23, pp. 5426-5429, 2009.

[24] Y. J. Rao, Z. L. Ran, X. Liao, and H. Y. Deng, "Hybrid LPFG/MEFPI sensor for simultaneous measurement of high-temperature and strain," Opt. Express, vol. 15, no. 22, pp. 14936-14941, 2007.

[25] Z. L. Ran, Y. Chen, Y. J. Rao, D. Sun, E. Lu, and Z. W. Liu, " $1100{ }^{\circ} \mathrm{C}$ fiber-optic high-temperature Fabry-Perot sensors fabricated by laser-micromachining," in Proc. SPIE, vol. 7753, pp. 775317, 2011

[26] Y. Gong, Y. J. Rao, Y. Guo, Z. L. Ran, and Y. Wu, "Temperature-insensitive micro Fabry-Pérot strain sensor fabricated by chemically etching Er-doped fiber," IEEE Photon. Technol. Lett., vol. 21, no. 22, pp. 1725-1727, 2009.

[27] Y. Gong, T. Zhao, Y. J. Rao, Y. Wu, and Y. Guo, “A ray-transfer-matrix model for hybrid fiber Fabry-Perot sensor based on graded-index multimode fiber," Opt. Express, vol. 18, no. 15, pp. 15844-15852, 2010

[28] Y. Gong, Y. Guo, Y. J. Rao, T. Zhao, and Y. Wu, "Fiber-optic Fabry-Perot sensor based on periodic focusing effect of graded-index multimode fibers," IEEE Photon. Tech. Lett., vol. 22, no. 23, pp. 1708-1710, 2010.

[29] Y. J. Rao, B. Xu, Z. L. Ran, and Y. Gong, "Micro extrinsic fiber-optic Fabry-Perot interferometric sensor based on Erbium and Boron doped fibers," Chin. Phys. Lett., vol. 27, no. 2, pp. 024208, 2010 (in Chinese).

[30] Y. Gong, Y. Guo, Y. J. Rao, T. Zhao, Y. Wu, and Z. L. Ran, "Sensitivity Analysis of Hybrid Fiber Fabry-Pérot Refractive-index Sensor," Acta Physica Sinica, vol. 60, no. 7, pp. 064202, 2011 (in Chinese).

[31] J. Jiang, Y. J. Rao, C. X. Zhou, and T. Zhu "Frequency-multiplexed fiber-optic Fizeau strain sensor system based on optical amplification," Acta Physica Sinica, vol. 53, no. 7, pp. 2221-2225, 2004 (in Chinese).

[32] Y. J. Rao, J. Jiang, and C. X. Zhou, "Spatial-frequency multiplexed fiber-optic Fizeau strain sensor system with optical amplification," Sens. Actuators, A: Physical, vol. 120, no. 2, pp. 354-359, 2005.

[33] Y. J. Rao, C. X. Zhou, and T. Zhu, "SFDM/CWDM 
of fiber-optic fizeau strain sensors," IEEE Photon. Technol. Lett., vol. 17, no. 6, pp. 1259-1261, 2005

[34] Y. J. Rao, X. J. Wang, T. Zhu, and C. X. Zhou, "Demodulation algorithm for spatial-frequencydivision-multiplexed fiber-optic Fizeau strain sensor networks," Opt. Lett., vol. 31, no. 6, pp. 700-702, 2006.

[35] Y. J. Rao "Recent progress in fiber-optic extrinsic Fabry-Perot interferometric sensors," Opt. Fiber Technol., vol. 12, no. 3, pp. 227-237, 2006.

[36] Y. J. Rao, "In-fiber Bragg grating sensors," Meas. Sci. Technol., vol. 8, no. 4, pp. 335-375, 1997.

[37] Y. J. Rao "Recent progress in applications of in-fiber Bragg grating sensors," Opt. Lasers Eng., vol. 31, no. 4, pp. 297-324, 1999.

[38] Y. J. Rao, D. A. Jackson, L. Zhang, and I. Bennion, "Dual-cavity interferometric wavelength-shift detection for in-fiber Bragg grating sensors," Opt. Lett., vol. 21, no. 19, pp 1556-1558, 1996.

[39] Y. J. Rao, D. A. Jackson, L. Zhang, and I. Bennion, "Extended dynamic range detection system for in-fiber Bragg grating strain sensors based on two cascaded interferometric wavelength scanners," Meas. Sci. Technol., vol. 8, no. 10, pp. 1043-1049, 1997.

[40] Y. J. Rao, M. R. Cooper, D. A. Jackson, C. N. Pannell, and L. Reekie, "Absolute strain measurement using an in-fiber-Bragg-grating-based Fabry-Perot sensor," Electron. Lett., vol. 36, no. 8, pp. 708-709, 2000.

[41] Y. J. Rao, M. R. Cooper, D. A. Jackson, C. N. Pannell, and L. Reekie, "Simultaneous measurement of displacement and temperature using in-fiber-Bragg-grating-based extrinsic Fizeau sensor," Electron. Lett., vol. 36, no. 19, pp. 1610-1612, 2000.

[42] Y. J. Rao, K. Kalli, G. Brady, D. J. Webb, D. A. Jackson, L. Zhang, and I. Bennion. "Spatially-multiplexed fiber-optic Bragg grating strain and temperature sensor system based on interferometric wavelength-shift detection," Electron. Lett., vol. 31, no. 12, pp. 1009-1010, 1995.

[43] Y. J. Rao, A. B. L. Ribeiro, D. A. Jackson, K. Kalli, L. Zhang, and I. Bennion, "Combined spatial- and time-division-multiplexing scheme for fiber grating sensors with drift-compensated phase-sensitive detection," Opt. Lett., vol. 20, no. 20, pp. 2149-2151, 1995.

[44] Y. J. Rao, A. B. L. Ribeiro, D. A. Jackson, L. Zhang, and I. Bennion, "In-fiber grating sensing network with a combined SDM, TDM, and WDM topology," in Conference Proceedings - Lasers and Electro-Optics Society Annual Meeting-LEOS, pp. 244, 1996.

[45] Y. J. Rao, A. B. L. Ribeiro, D. A. Jackson, L. Zhang, and I. Bennion, "Simultaneous spatial, time and wavelength division multiplexed in-fiber grating sensing network," Opt. Commun., vol. 125, no. 1-3, pp. 53-58, 1996.
[46] Y. J. Rao, D. J. Webb, D. A. Jackson, L. Zhang, and I. Bennion, "High-resolution, wavelength-divisionmultiplexed in-fiber Bragg grating sensor system," Electron. Lett., vol. 32, no. 10, pp. 924-926, 1996.

[47] Y. J. Rao, D. A. Jackson, L. Zhang, and I. Bennion, "Strain sensing of modern composite materials with a spatial/wavelength-division multiplexed fiber grating network," Opt. Lett., vol. 21, no. 9, pp. 683-685, 1996.

[48] Y. J. Rao, P. J. Henderson, D. A. Jackson, L. Zhang, and I. Bennion, "Simultaneous strain, temperature and vibration measurement using a multiplexed in-fiber-Bragg-grating/fiber-Fabry-Perot sensor system," Electron. Lett., vol. 33, no. 24, pp. 2063-2064, 1997.

[49] Y. J. Rao, D. J. Webb, D. A. Jackson, L. Zhang, and I. Bennion, "In-fiber Bragg-grating temperature sensor system for medical applications," $J$. Lightwave Technol., vol. 15, no. 5, pp. 779-785, 1997.

[50] Y. J. Rao, D. J. Webb, D. A. Jackson, L. Zhang, and I. Bennion, "Optical in-fiber Bragg grating sensor systems for medical applications," J. Biomed. Opt. vol. 3, no. 1, pp. 38-44, 1998.

[51] Y. J. Rao, S. F. Yuan, X. K. Zeng, D. K. Lian, Y. Zhu, Y. P. Wang, S. L. Huang, T. Y. Liu, G. F. Fernando, L. Zhang, and I. Bennion "Simultaneous strain and temperature measurement of advanced 3-D braided composite materials using an improved EFPI/FBG system," Opt. Lasers Eng., vol. 38, no. 6, pp. 557-566, 2002

[52] Y. J. Rao, Z. L. Ran, and C. X. Zhou, "Fiber-optic Fabry-Perot sensors based on a combination of spatial-frequency division multiplexing and wavelength division multiplexing formed by chirped fiber Bragg grating pairs," Appl. Opt., vol. 45, no. 23, pp. 5815-5818, 2006.

[53] Y. J. Rao, Z. L. Ran, and R. R. Chen, "Long-distance fiber Bragg grating sensor system with a high optical signal-to-noise ratio based on a tunable fiber ring laser configuration," Opt. Lett., vol. 31, no. 18, pp. 2684-2686, 2006.

[54] Y. J. Rao, S. Feng, Q. Jiang, and Z. L. Ran, "Ultra-long distance $(300 \mathrm{~km})$ fiber Bragg grating sensor system using hybrid EDF and Raman amplification," in Proc. SPIE, The International Society for Optical Engineering, 20th International Conference on Optical Fiber Sensors, vol. 7503, pp. 75031Q, 2009.

[55] Z. L. Ran and Y. J. Rao "A FBG sensor system with cascaded LPFGs and Music algorithm for dynamic strain measurement," Sens. Actuators, A: Physical, vol. 135, no. 2, pp. 415-419, 2007.

[56] H. J. Wu, Y. J. Rao, C. Tang, Y. Wu, and Y. Gong, “A novel FBG-based security fence enabling to detect extremely weak intrusion signals from nonequivalent sensor nodes," Sens. Actuators, A: Physical, vol. 167, no. 2 , pp. 548-555, 2011. 